\section{Integrated high-speed digital optical true-time-delay line with embedded electrically switchable gratings}

\author{
Xuejun Lu \\ University of Massachusetts Lowell \\ One University Avenue \\ Lowell, Massachusetts 01854 \\ E-mail: xuejun_lu@uml.edu \\ Suning Tang \\ Jibing Lin \\ Jennifer Cologrove \\ Yuanji Tang \\ X. Wang \\ Crystal Research, Inc. \\ 48501 Warm Springs Boulevard \\ Fremont, California 94539
}

\section{Lei Zheng}

University of Massachusetts Lowell

One University Avenue

Lowell, Massachusetts 01854

\section{James Foshee}

Air Force Research Laboratory

Platform Connectivity Branch (IFGD)

Wright-Patterson Air Force Base, Ohio 45433

\begin{abstract}
A monolithically integrated high-speed true-timedelay line with embedded electrically switchable Bragg gratings was developed and characterized. Such a monolithically integrated device structure leads to a significantly reduced device size and allows a more precise RF phase control. A 40 ps reprogrammable optical true-time delay increment was acheived with a very fast switching speed of $<50 \mu \mathrm{s}$. (๑) 2005 Society of Photo-Optical Instrumentation Engineers. [DOI: 10.1117/1.1906208]
\end{abstract}

Subject terms: polymeric waveguides; electro-optics; holographic polymer dispersed liquid crystals; optical true-time-delay.

Paper 050014L received Jan. 14, 2005; revised manuscript received Feb. 22, 2005; accepted for publication Feb. 24, 2005; appeared online Mar. 2, 2005; published online May 26, 2005.

\section{Introduction}

Optical true-time-delay (TTD) techniques are expected to provide frequency-independent time delays over a wide operation bandwidth and thus enables multiband (UHF, P, L, $\mathrm{X}$, and Ku-bands) squint-free beam steering for phase array antenna (PAA) and synthetic aperture radar (SAR) applications. ${ }^{1,5}$ A variety of optical time-delay technologies have been developed, including detector-switched optical waveguide delay lines, ${ }^{2}$ fiber-grating prism based wavelength tunable laser diodes ${ }^{3}$ and $\mathrm{LiNbO}_{3}$ switches over fiber distribution networks. ${ }^{4}$

0091-3286/2005/\$22.00 @ 2005 SPIE
In this paper, we present a monolithically integrated optical waveguide true-time-delay line with embedded highspeed electrically switchable Bragg gratings based on polymer-dispersed liquid crystals (LC). ${ }^{6}$ We have demonstrated (1) 16-cm-long polymeric waveguides with low propagation loss $(0.2 \mathrm{~dB} / \mathrm{cm})$, (2) electrically switchable Bragg gratings with high switching speed $(<50 \mu \mathrm{s})$ and large diffraction efficiency ( $>99 \%$ ), and (3) precisely digital controllable time delays with 40-ps increments.

\section{Device Physics}

The digital optical true-time-delay line, shown in Fig. 1, consists of a $16 \mathrm{~cm}$-long polymeric channel waveguide fabricated on a $4 \mathrm{~cm}$ long silicon substrate. The polymeric channel waveguide has a $6 \times 6 \mu \mathrm{m}$ cross section and an effective refractive index of 1.575 . It is sandwiched between top and bottom polymeric cladding layers with a refractive index of 1.562 . The polymeric channel waveguide contains holographically defined Bragg grating structures with polymer-dispersed liquid crystal (LC) nanocomposites (HPDLC). The fabrication of HPDLC has been presented elsewhere. ${ }^{7}$ The orientation of the LC molecules can be electrically aligned by an external electric field. The refractive indices of randomly oriented LC and electrically aligned LC (for TM polarized light beam) are $n_{0}=1.561$ and $n_{c}=1.777$, respectively. The top cladding is covered with patterned electrodes to apply an electric field for electro-optical orientations.

The mechanism of the electrically switchable Bragg grating (ESBG) is based on the index matching and mismatching between the liquid crystal region and the polymer region in the Bragg gratings formed by holography technology. Since the refractive indices are different for different LC orientations, an electric field aligns the LC directors and thus changes the refractive indices in droplets but does not affect surrounding polymers, which results in switchable Bragg gratings controlled by an external electric field. ${ }^{6}$ The electrically switchable Bragg grating is a volume phase grating with well-predicted diffraction properties by the Kogelnik model. ${ }^{8}$ Since the diameters of the liquid crystal droplets $(\sim 100 \mathrm{~nm})$ are considerably less than the operating wavelength of light (600 to $1600 \mathrm{~nm}$ ), the clouds of droplets are "seen" as a homogeneous region with an average index $\left(n_{\mathrm{LCM}}\right)$ different from that of the interspersed polymer regions $\left(n_{p}\right)$. The diffraction properties of an ESBG can be modeled by assuming an index modulation given by:

$n=n_{0}+n_{1} \cos (\vec{K} \cdot \vec{r})$,

where $n_{0}$ is the average refractive index, $\vec{K}$ is the grating vector, and $\vec{r}$ is a position coordinate. In Eq. (1), $n_{1}$ is the index modulation amplitude given by

$n_{1}=\left(2 f_{c} / \pi\right)\left(n_{L C}-n_{p}\right) \sin [\alpha \pi]$,

where $f_{c}$ is the volume fraction of the phase-separated liquid crystal in the ESBG, $n_{p}$ is the polymer index, and $\alpha$ is the fraction of grating period $\Lambda$ occupied by the liquid crystal droplets. By properly controlling $f_{c}$ and $\alpha$, a refractive index modulation amplitude of $\sim 0.1$ can be achieved.

For an in-line reflection hologram, the equations of diffraction efficiency for TE and TM polarizations are identical to that predicted by Kogelnik as well, given as ${ }^{8}$ 
$\mathbf{E}$

$\mathbf{L}$

E

T

$\mathrm{T}$

E

$\mathbf{R}$

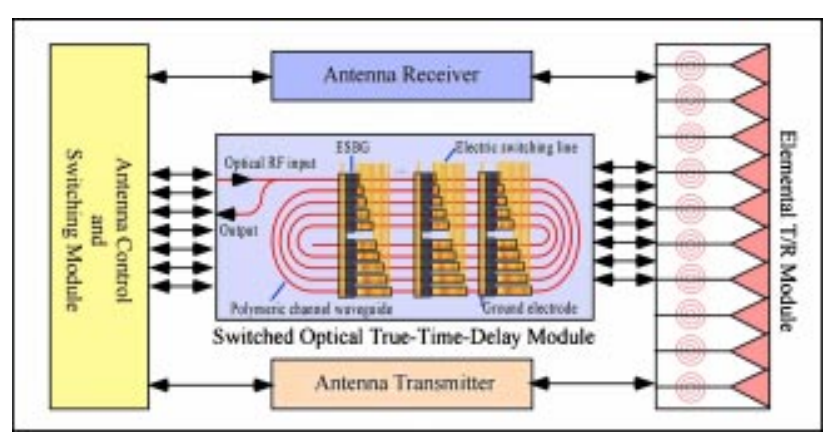

Fig. 1 Electrical block diagram of a photonic wideband phased array using switched optical waveguide true-time-delay lines.

$R=\tanh ^{2}\left[\pi n_{1} d / \lambda\right]$,

where $R$ is the peak reflection, $d$ is the grating length, and $\lambda$ is the Bragg wavelength. The electrically switchable Bragg gratings fabricated have a grating length $d=30 \mu \mathrm{m}$ and $n_{1}=0.02$ at a low switching voltage of $20 \mathrm{~V}$. A reflection efficiency $>99 \%$ was obtained.

Since each grating can be electrically switched on-andoff to-allow the signal to either reflect or bypass the waveguide, an array of delay times $T$ can be generated at any value at an increment of $2 \Delta \tau$, up to the maximum value $T_{\max }=N *(2 \Delta \tau)$, where $N$ is the number of individual gratings, $\Delta \tau=n L / c, L$ is the spatial separation between every two gratings, $n$ is the effective index of waveguide, and $c$ is the speed of light in a vacuum. A polymeric waveguide delay line with 4-mm separations between the embedded switchable Bragg gratings was developed, which corresponds to 40-ps optical time-delay increments.

\section{Device Characterization}

The performance of the TTD is characterized employing a mode-locked fiber (Calmar FPL-01) laser system, which generates narrow optical pulses $(<10 \mathrm{ps})$ that were subsequently launched into the TTD line. The reflected output signals are routed through a fiber-optic circulator and sequentially sampled by a digital sampling oscilloscope. The whole system was synchronized by the trigger system.Characterization of the setup can be found elsewhere. ${ }^{7}$

When the electrical switch control unit turns on the electrically switchable Bragg gratings in the delay-line waveguide, a reflected optical signal is displayed on the sampling scope at "zero" position. If the electrical switch control unit turns on another electrically switchable Bragg grating, the second reflected pulse will show on the scope screen at a different position, corresponding to a true time delay of $\sim 40 \mathrm{ps}$, which is in line with the round trip distance between the two electrically switchable Bragg gratings $(4 \mathrm{~mm})$ on the waveguide delay-line module. By switching on different gratings, various true time delays can be readily obtained. Figure 2 illustrates $40-p s$ time delay intervals, corresponding to the 4-mm separations of the three gratings in the polymeric waveguide delay line. In Fig. 2, the secondary peaks following the main reflected pulse are due to the narrow reflection bandwidth of the switchable Bragg grating, which, when transferred into time domain, presents a sinc function profile. Detailed analysis can be found in Ref. 9 .

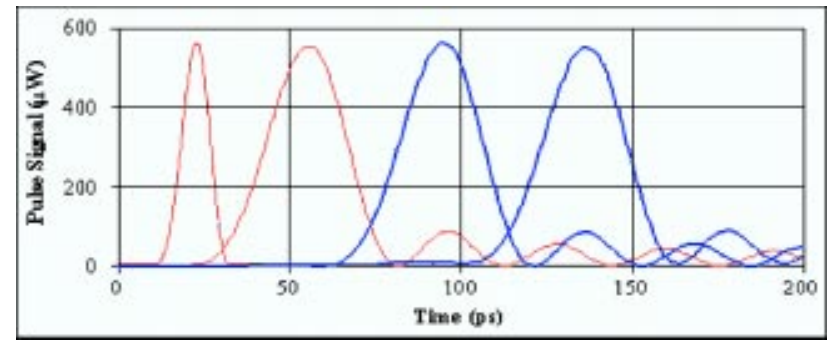

Fig. 2 Demonstration of 40-, 80-, and 120-ps optical true time delays by switching on the first three gratings with separations of 4,8 , and $12 \mathrm{~mm}$, respectively, in the polymeric waveguide delay line. The input pulse is located at $22 \mathrm{ps}$.

The switching speed of the switchable Bragg gratings was characterized by sending a cw laser into the waveguide and measuring the time delay between the electrical switch-on signal and the returned optical signals. Since the propagation time of the optical signal in the waveguide is much shorter $(\sim 40 \mathrm{ps})$ than the grating response time $(\mu \mathrm{s})$, the delay is essentially the response time of the electrically switchable Bragg gratings, which was measured to be 50 $\mu \mathrm{s}$. The insertion losses were measured to be $0.3,0.4$, and $0.5 \mathrm{~dB}$ for the first three Bragg gratings, respectively, when they were individually switched on.

\section{Conclusion}

We have successfully demonstrated an integrated optical true-time-delay line based on ultra-low-loss polymeric waveguides and electrically switchable Bragg gratings. A reflection efficiency $>99 \%$ was obtained at a low driving voltage of $20 \mathrm{~V}$ with a very fast switching speed $(<50 \mu$ s) that is at least 100 times faster than any existing commercial optical switch. Reprogrammable optical true time delays of 40 ps were achieved. The linear phase-frequency relations were verified over a wideband frequency range.

\section{Acknowledgment}

This research is supported by AFRL and NASA.

\section{References.}

1. N. A. Riza, Ed., Selected Papers on Photonic Control Systems for Phased Array Antennas, SPIE Milestone Series, Vol. MS 136, SPIE Press, Bellingham, WA (1997).

2. S. Tang, R. T. Chen, and J. Foshee, "Polymeric waveguide circuits for airborne photonic phased array antennas," IEEE Circuits and Devices 16(1), 10-16 (2000).

3. H. Zmuda, R. A. Soref, P. Payson, S. Johns, and E. N. Toughlian, "Photonic beamformer for phased array antennas using a fiber grating prism," IEEE Photonics Technol. Lett. 9(2), 241-243 (1997).

4. E. J. Murphy, T. F. Adda, W. J. Minford, R. W. Irin, E. I. Ackerman, and S. B. Adams, "Guided-wave optical time delay network," IEEE Photonics Technol. Lett. 8(4), 545-547 (1996).

5. A. P. Goutzoulis and R. P. Gouse, "Comparison of conventional and fiber optic manifolds for a dual band (UHF and S) phased-array antenna," IEEE Trans. Antennas Propag. 45(2), 246-253 (1997).

6. T. J. Bunning, L. V. Natarajan, V. P. Tondiglia, and R. L. Sutherland, "Switchable holographic gratings formed using polymer dispersed liquid crystals," Proc. IEEE Lasers and Electro-Optics Society Annual Meeting 1, 358-359 (1996).

7. J. Foshee, J. Colegrove, Y. Tang, Z. Shi, X. Zhang, and S. Tang, "Switched optical polymeric waveguide true-time-delay lines for wideband photonics phased array antennas," Proc. SPIE 5356, 65-73 (2004).

8. H. Kogelnik, "Coupled wave theory for thick hologram gratings," Bell Syst. Tech. J. 48, 2909-2947 (1969).

9. L. Dong and S. Fortier, "Formulation of time-domain algorithm for fiber Bragg grating simulation and reconstruction," IEEE J. Quantum Electron. 40, 1087-1098 (2004). 